International Mathematical Forum, Vol. 8, 2013, no. 1, 1 - 8

\title{
Consistent FE-Analysis of Strang's Problem Based on Mixed Formulations
}

\author{
D. Hage, J. Frohne and F. T. Suttmeier ${ }^{1}$ \\ Department Mathematik \\ Universität Siegen \\ D-57258 Siegen, Germany
}

\begin{abstract}
In this paper our studies on techniques for a posteriori error control and adaptive mesh design for finite element models in perfect plasticity are continued. The focus is on the numerical analysis of a low-order, dual-mixed discretisation. A posteriori error estimates are provided. Numerical tests confirm the theoretical results.
\end{abstract}

Keywords: adaptive finite elements, a posteriori error-bounds, mixed methods, variational inequality, anti-plane shear problem

\section{Introduction}

The work at hand is devoted to the numerical treatment of systems of partial differential equations, where the solution is subjected to inequality constraints. We employ the finite element Galerkin (FE) method to obtain approximate solutions of such systems.

The basis for applying an FE discretisation is a suitable mathematical setting, which in the topics under consideration takes the form of variational inequalities (VI). An introduction to mathematical issues of these topics can be found in Kinderlehrer \& Stampacchia [9] and Duvaut \& Lions [3]. A corresponding overview on numerical methods is given in Glowinski et. al. [5, 4].

Variational inequality settings typically arise in the field of continuum mechanics. Examples are contact problems, where the displacement is restricted by a rigid obstacle or plastic materials, where certain norms of the stresses are bounded.

From mathematical point of view elasto-plastic problems are optimisation problems, where the stress field is subjected to inequality constraints which have to be fulfilled in the pointwise sense. A first straight-forward approach for

\footnotetext{
${ }^{1}$ suttmeier@mathematik.uni-siegen.de
} 
a corresponding FE-scheme starts from the primal-mixed formulation. Here, the stresses can be eliminated and then the problem can be written by means of a projection onto the yield surface as an nonlinear equality in terms of derivatives of the displacement field $u$.

The drawback of this formulation is, that even very simple examples in the elasto-plastic case show, that discontinuities in the displacement $u$ may occur (see Suquet [12]) due to slip lines in the micro-structure, whereas the stresses $\sigma$, determined by linear combinations of derivatives of $u$, have a smoother behaviour. For example in Bensoussan \& Frehse [2] and Seregin [10] there is proven, that the stresses belong to $H_{l o c}^{1}$.

Due to these regularity results it seems to be more adequate to consider the dual-mixed approach since in this case derivatives of $u$ are not entering this formulation. Furthermore, from a practical point of view, the stresses are required with high accuracy. Therefore dual-mixed methods treating the stresses directly are often more adequate for solving problems in continuum mechanics.

\section{Model problem}

In the following, we present our ideas at the anti-plane shear or Strang's example. (c.f. Strang [11]). The mathematical problem seeks for a scalar displacement $u$ and a stress vector $\sigma=\left(\sigma_{1}, \sigma_{2}\right)$ as functions on a bounded domain $\Omega \subset \mathbb{R}^{2}$. Stresses and displacement are caused by an external load described by a scalar function $f$ on $\Omega$. A nonlinear relation between $u$ and $\sigma$ - the so called plastic behaviour of the material - is taken into account by the restriction $|\sigma| \leq 1$. The classical form of the whole system is given by

$$
\begin{gathered}
-\operatorname{div} \sigma=f, \quad \sigma=\Pi \nabla u \quad \text { in } \Omega, \\
u=0 \quad \text { on } \partial \Omega,
\end{gathered}
$$

where $\Pi$ denotes the pointwise projection onto the circle with radius 1 .

In order to give a weak form for (1), we set

$$
\begin{aligned}
H & :=\left\{\tau \in L^{2}\left(\Omega, \mathbb{R}^{2}\right) \mid \operatorname{div} \tau \in L^{2}(\Omega)\right\}, \\
\Pi H & :=\{\tau \in H,|\tau|-1 \leq 0\}, \\
V & :=\left\{\varphi \in L^{2}(\Omega)\right\},
\end{aligned}
$$

where $|\tau|^{2}=\tau_{1}^{2}+\tau_{2}^{2}$. Now, similar to the approach in Johnson [7], the solution $\{\sigma, u\} \in \Pi H \times V$ is determined by the variational inequality

$$
(\sigma, \tau-\sigma)+(u, \operatorname{div}(\tau-\sigma))-(\operatorname{div} \sigma, \varphi) \geq(f, \varphi) \quad \forall\{\tau, \varphi\} \in \Pi H \times V
$$

where $(.,$.$) denotes the L^{2}$-scalar product on $H$. Existence of the solution and uniquenes for the stresses $\sigma$ have been proven, e.g., by Johnson [6]. From 
regularity theory (see e.g. Seregin [10]) one knows (roughly spoken) the stresses to fulfill $\sigma \in\left(H^{1}(\Omega)\right)^{2}$.

The discrete analogue seeks for a pair $\left\{\sigma_{h}, u_{h}\right\}$ fulfilling

$$
\begin{aligned}
\left(\sigma_{h}, \tau-\sigma_{h}\right)+ & \left(u_{h}, \operatorname{div}\left(\tau-\sigma_{h}\right)\right) \\
& \left(-\operatorname{div} \sigma_{h}, \varphi\right)+\delta_{h}\left(\left[u_{h}\right],[\varphi]\right) \geq(f, \varphi) \forall\{\tau, \varphi\} \in \Pi H_{h} \times V_{h} .
\end{aligned}
$$

The finite dimensional space $\Pi H_{h} \times V_{h}$ for a discretisation on triangulations $\mathbb{T}_{h}$ with mesh size parameter $h$ only consisting of triangular elements $T$ for this saddle point problem is determined by approximating each component of the stresses by the standard linear shape functions for $\Pi H_{h}$. $V_{h}$ is constructed by elementwise constant functions. Above the brackets [.] denote the jump across the element boundaries $\partial T$ and $\delta_{h}$ is an appropriatly chosen mesh-dependent parameter. For details to this notation we refer to Becker [1].

\section{Error control}

In order to provide an error estimate for $\left\|\sigma-\sigma_{h}\right\|$, we introduce - following Johnson e.a. $[6,8]$ - the regularised version with parameter $\mu>0$ of (2) in the form

$$
\begin{aligned}
& \left(\sigma_{\mu}, \tau\right)+\left(\mu^{-1}\left(\sigma_{\mu}-\Pi \sigma_{\mu}\right), \tau\right) \\
& \quad+\left(u_{\mu}, \operatorname{div} \tau\right)-\left(\operatorname{div} \sigma_{\mu}, \varphi\right)=(f, \varphi) \quad \forall\{\tau, \varphi\} \in H \times V .
\end{aligned}
$$

In view of

$$
\left\|\sigma-\sigma_{h}\right\| \leq\left\|\sigma-\sigma_{\mu}\right\|+\left\|\sigma_{\mu}-\sigma_{h}\right\|
$$

it remains to provide seperate estimates for $\left\|\sigma-\sigma_{\mu}\right\|$ and $\left\|\sigma_{\mu}-\sigma_{h}\right\|$, which will be done now.

\subsection{A priori estimate}

Here, we focus on the estimate for $\left\|\sigma-\sigma_{\mu}\right\|$. The computation is as follows. Exploiting monotonicity of the regularisation and recalling that by construction there holds $|\sigma| \leq 1$, we get

$$
(\mu^{-1}\left(\sigma_{\mu}-\Pi \sigma_{\mu}\right)-\mu^{-1} \overbrace{(\sigma-\Pi \sigma)}^{=0}, \sigma_{\mu}-\sigma) \geq 0 .
$$

Additionally one employs $(-\operatorname{div} \sigma, \varphi)=\left(-\operatorname{div} \sigma_{\mu}, \varphi\right)=(f, \varphi)$, yielding

$$
\begin{gathered}
\left\|\sigma_{\mu}-\sigma\right\|^{2} \leq\left(-\sigma+\sigma_{\mu}, \sigma_{\mu}-\sigma\right)+\left(\mu^{-1}\left(\sigma_{\mu}-\Pi \sigma_{\mu}\right)-\mu^{-1}(\sigma-\Pi \sigma), \sigma_{\mu}-\sigma\right) \\
=-\left(\sigma, \sigma_{\mu}-\sigma\right)+\left(\sigma_{\mu}, \sigma_{\mu}-\sigma\right) \\
\quad+\left(\mu^{-1}\left(\sigma_{\mu}-\Pi \sigma_{\mu}\right), \sigma_{\mu}-\sigma\right)+\left(u_{\mu}, \operatorname{div}\left(\sigma_{\mu}-\sigma\right)\right) .
\end{gathered}
$$


With (4) it remains

$$
\left\|\sigma_{\mu}-\sigma\right\|^{2}=-\left(\sigma, \sigma_{\mu}-\sigma\right)=0 \quad \text { for } \mu \rightarrow 0,
$$

where the limit process is ensured by the weak convergence of $\sigma_{\mu}$ (see Johnson $[6])$.

\subsection{A posteriori estimate}

Here, we focus on the estimate for $\left\|\sigma_{\mu}-\sigma_{h}\right\|$. Arguing as above the computation starts with

$$
\begin{aligned}
& \left(\sigma_{\mu}-\sigma_{h}, \sigma_{\mu}-\sigma_{h}\right)+\delta_{h}\left(\left[u_{\mu}-u_{h}\right],\left[u_{\mu}-u_{h}\right]\right) \\
& \leq\left(\sigma_{\mu}, \sigma_{\mu}-\sigma_{h}\right)+\left(u_{\mu}, \operatorname{div} \sigma_{\mu}-\operatorname{div} \sigma_{h}\right)+\underbrace{(\mu^{-1}\left(\sigma_{\mu}-\Pi \sigma_{\mu}\right)-\mu^{-1} \overbrace{\left(\sigma_{h}-\Pi \sigma_{h}\right)}^{=0}, \sigma_{\mu}-\sigma_{h})}_{\geq 0, \text { monotone operator }} \\
& \quad-\left(u_{\mu}-u_{h}, \operatorname{div} \sigma_{\mu}-\operatorname{div} \sigma_{h}\right)+\delta_{h}\left(\left[u_{\mu}-u_{h}\right],\left[u_{\mu}-u_{h}\right]\right) \\
& \quad-\left(u_{h}, \operatorname{div} \sigma_{\mu}-\operatorname{div} \sigma_{h}\right)-\left(\sigma_{h}, \sigma_{\mu}-\sigma_{h}\right)
\end{aligned}
$$

Again using (4) the first terms vanish and it remains

$$
\left(\sigma_{\mu}-\sigma_{h}, \sigma_{\mu}-\sigma_{h}\right)+\delta_{h}\left(\left[u_{\mu}-u_{h}\right],\left[u_{\mu}-u_{h}\right]\right) \leq T 1+T 2
$$

with

$$
\begin{aligned}
& T 1=-\left(u_{\mu}-u_{h}, \operatorname{div} \sigma_{\mu}-\operatorname{div} \sigma_{h}\right)+\delta_{h}\left(\left[u_{\mu}-u_{h}\right],\left[u_{\mu}-u_{h}\right]\right) \\
& T 2=-\left(u_{h}, \operatorname{div} \sigma_{\mu}-\operatorname{div} \sigma_{h}\right)-\left(\sigma_{h}, \sigma_{\mu}-\sigma_{h}\right) .
\end{aligned}
$$

\subsubsection{Estimation of $T 1$}

Below the standard interpolant of $u_{\mu}$ will be denoted by $u_{i}$. Next we observe that for arbitrary $\varphi_{h} \in V_{h}$ there holds

$$
\begin{aligned}
\left(\varphi_{h}, \operatorname{div} \sigma_{\mu}-\operatorname{div} \sigma_{h}\right) & =-\left(f, \varphi_{h}\right)+\left(f, \varphi_{h}\right)-\delta_{h}\left(\left[u_{h}\right],\left[\varphi_{h}\right]\right) \\
& =-\delta_{h}\left(\left[u_{h}\right],\left[\varphi_{h}\right]\right) .
\end{aligned}
$$

Now we obtain the identity

$$
\begin{aligned}
-\left(u_{\mu}-u_{h}, \operatorname{div} \sigma_{\mu}-\operatorname{div} \sigma_{h}\right) & +\delta_{h}\left(\left[u_{h}\right],\left[u_{h}\right]\right) \\
& =-\left(u_{\mu}-u_{i}, \operatorname{div} \sigma_{\mu}-\operatorname{div} \sigma_{h}\right)+\delta_{h}\left(\left[u_{h}\right],\left[u_{i}\right]\right) .
\end{aligned}
$$

Here, using interpolation estimates found in Johnson \& Hansbo [8], the first term on the right-hand-side can be estimated by

$$
\begin{aligned}
\left(u_{\mu}-u_{i}, \operatorname{div} \sigma_{\mu}-\operatorname{div} \sigma_{h}\right) & =\sum_{T}\left(u_{\mu}-u_{i}, f-f_{i}\right)_{T} \\
& \leq c \sum_{T}\left\|h_{T}\left(f-f_{i}\right)\right\|_{\infty, T}\left\|\nabla u_{\mu}\right\|_{L_{1}(T)} .
\end{aligned}
$$


Analogously the jumps can be controlled by

$$
\begin{aligned}
\delta_{h}\left(\left[u_{h}\right],\left[u_{i}\right]\right)_{T} & \leq \delta_{h}\left(\left[u_{h}\right], u_{i}^{l}-u_{\mu}\right)_{\partial T}+\delta_{h}\left(\left[u_{h}\right], u_{\mu}-u_{i}^{r}\right)_{\partial T} \\
& \leq c \delta_{h}\left\|h_{T} D_{h} u_{h}\right\|_{\infty, T}\left\|\nabla u_{\mu}\right\|_{L_{1}(T)}
\end{aligned}
$$

where $u_{i}^{l}$ and $u_{i}^{r}$ denote the values of $u_{i}$ on the two triangles having an edge of $\partial T$ in common and according to the notation introduced in Johnson \& Hansbo [8] we set

$$
D_{h} u_{h}=\max _{\partial T} \frac{\left|\left[u_{h}\right]\right|}{h_{T}} .
$$

Under the assumption of the so-called safe load hypothesis (see e.g. Johnson [6]) $\left\|\nabla u_{\mu}\right\|_{L_{1}}$ is - uniformly with respect to $\mu$ - bounded. In this note in practice this norm is approximately evaluated by exploiting values of $D_{h} u_{h}$.

\subsubsection{Estimation of $T 2$}

In order to tackle the estimation of $T 2$ we introduce the auxiliary problem of finding $u_{L}$ fulfilling

$$
\begin{gathered}
\left(\frac{1}{p\left(u_{L}\right)} \nabla u_{L}, \varphi_{L}\right)=\left(\sigma_{h}, \nabla \varphi_{L}\right) \quad \forall \varphi_{L} \in V_{L} \\
p\left(u_{L}\right)= \begin{cases}1, & \left|\nabla u_{L}\right| \leq 1 \\
\left|\nabla u_{L}\right|, & \text { otherwise. }\end{cases}
\end{gathered}
$$

Here the discrete space $V_{L} \subset H_{0}^{1}(\Omega)$ is defined on $\mathbb{T}_{h}$ using the standard linear shape functions and $H_{0}^{1}$ is a standard Sobolev space including zero boundary conditions. In fact this is a discrete version of a primal formulation for our plasticity problem, where the external load is determined by information namely $\sigma_{h}$ - of the discrete dual-mixed setting.

Now we can estimate $T 2$ as follows.

$$
\begin{aligned}
&-\left(u_{h},\right.\left.\operatorname{div} \sigma_{\mu}-\operatorname{div} \sigma_{h}\right)-\left(\sigma_{h}, \sigma_{\mu}-\sigma_{h}\right) \\
&=-\left(u_{h}-u_{L}, \operatorname{div} \sigma_{\mu}-\operatorname{div} \sigma_{h}\right)-\left(u_{L}, \operatorname{div} \sigma_{\mu}-\operatorname{div} \sigma_{h}\right)-\left(\sigma_{h}, \sigma_{\mu}-\sigma_{h}\right) \\
&=\left(u_{h}-u_{L}, f+\operatorname{div} \sigma_{h}\right)+\left(\nabla u_{L}-p\left(u_{L}\right) \sigma_{h}, \sigma_{\mu}-\sigma_{h}\right)+\left(\lambda_{L} \sigma_{h}, \sigma_{\mu}-\sigma_{h}\right) \\
& \leq\left(u_{h}-u_{L}, f+\operatorname{div} \sigma_{h}\right)+\frac{1}{2}\left\|\nabla u_{L}-p\left(u_{L}\right) \sigma_{h}\right\|^{2} \\
& \quad+\frac{1}{2}\left\|\sigma_{\mu}-\sigma_{h}\right\|^{2}+\int_{\Omega} \lambda_{L}\left|\sigma_{h}\right|\left(1+C \mu^{1 / 2}-\left|\sigma_{h}\right|\right) d x
\end{aligned}
$$

where $\lambda_{L}$ is given by $\lambda_{L}=p\left(u_{L}\right)-1$ and $\mu^{-1}\left|\sigma-\sigma_{\mu}\right|^{2} \leq C$ (see Johnson [6]) is used. 


\subsection{Error bound}

Eventually collecting terms from subsections above and passing to the limit $\mu \rightarrow 0$ we obtain

Theorem 2.1. With notations introduced above there holds an estimate for the error between the solutions $\sigma$ of (2) an $\sigma_{h}$ of (3) in the form

$$
\left\|\sigma-\sigma_{h}\right\|^{2} \leq \sum_{T}\left(\rho_{u}^{2}+\rho_{\sigma}^{2}+\rho_{p}^{2}+\rho_{j}^{2}\right)
$$

with the settings

$$
\begin{array}{ll}
\rho_{u}^{2}=\left(u_{h}-u_{L}, f+\operatorname{div} \sigma_{h}\right), & \rho_{\sigma}^{2}=\frac{1}{2}\left\|\nabla u_{L}-p\left(u_{L}\right) \sigma_{h}\right\|^{2}, \\
\rho_{p}^{2}=\int_{\Omega} \lambda_{L}\left|\sigma_{h}\right|\left(1-\left|\sigma_{h}\right|\right) d x, & \rho_{j}^{2}=\delta_{h}\left(\left[u_{h}\right],\left[u_{h}\right]\right)_{T} .
\end{array}
$$

\section{Numerical results}

As a simple test we choose the second example proposed in Johnson \& Hansbo [8]. The evaluation of the single components of our error estimate, determined on a sequence of regular refined meshes are shown in Table 1. Here one observes the jumps of $u_{h}$ measured by $\rho_{j}$ to be dominant.

Furthermore assuming the components to behave like $\mathcal{O}\left(h^{\alpha}\right)$ we determine an approximation $\alpha_{(.)}$on each level, by comparing the error bounds with the estimator on the previous level. The results are listed in Table 2. One observes $\alpha_{(.)}$tending towards 1 , indicating the components of the estimate to behave like $\mathcal{O}(h)$, as one would anticipate. Again $\rho_{j}$ turns out to be the critical term.

Eventually a sequence of adaptively refined grids, generated on the basis of the weighted estimate, is depicted in Figure 1. Especially the critical zone where plastification occurs is well resolved. The background colour is given by values of $p^{-1}\left(u_{L}\right)$.

\begin{tabular}{rcccc}
\hline Cells & $\rho_{\sigma}$ & $\rho_{u}$ & $\rho_{p}$ & $\rho_{\text {jumps }}$ \\
\hline 32 & $2.349925 \mathrm{e}-01$ & $2.048310 \mathrm{e}-01$ & $5.014007 \mathrm{e}-02$ & $2.046361 \mathrm{e}-01$ \\
128 & $1.315747 \mathrm{e}-01$ & $1.104788 \mathrm{e}-01$ & $2.910051 \mathrm{e}-02$ & $1.712313 \mathrm{e}-01$ \\
512 & $6.939553 \mathrm{e}-02$ & $5.516487 \mathrm{e}-02$ & $1.613156 \mathrm{e}-02$ & $1.055047 \mathrm{e}-01$ \\
2048 & $3.467687 \mathrm{e}-02$ & $2.495607 \mathrm{e}-02$ & $9.385640 \mathrm{e}-03$ & $5.952007 \mathrm{e}-02$ \\
8192 & $1.744209 \mathrm{e}-02$ & $1.134331 \mathrm{e}-02$ & $4.539062 \mathrm{e}-03$ & $3.134596 \mathrm{e}-02$ \\
32768 & $8.751919 \mathrm{e}-03$ & $5.082170 \mathrm{e}-03$ & $2.304256 \mathrm{e}-03$ & $1.620830 \mathrm{e}-02$ \\
\hline
\end{tabular}

Table 1: Computational results for the test example. Evaluation of the single components of our error estimate, determined on a sequence of regular refined meshes. 


\begin{tabular}{rcccc}
\hline Cells & $\alpha_{\sigma}$ & $\alpha_{u}$ & $\alpha_{p}$ & $\alpha_{\text {jumps }}$ \\
\hline 32 & $0.000000 \mathrm{e}+00$ & $0.000000 \mathrm{e}+00$ & $0.000000 \mathrm{e}+00$ & $0.000000 \mathrm{e}+00$ \\
128 & $8.367326 \mathrm{e}-01$ & $8.906645 \mathrm{e}-01$ & $7.849196 \mathrm{e}-01$ & $2.571142 \mathrm{e}-01$ \\
512 & $9.229675 \mathrm{e}-01$ & $1.001948 \mathrm{e}+00$ & $8.511585 \mathrm{e}-01$ & $6.986392 \mathrm{e}-01$ \\
2048 & $1.000869 \mathrm{e}+00$ & $1.144359 \mathrm{e}+00$ & $7.813589 \mathrm{e}-01$ & $8.258591 \mathrm{e}-01$ \\
8192 & $9.914008 \mathrm{e}-01$ & $1.137549 \mathrm{e}+00$ & $1.048061 \mathrm{e}+00$ & $9.250967 \mathrm{e}-01$ \\
32768 & $9.949016 \mathrm{e}-01$ & $1.158325 \mathrm{e}+00$ & $9.780932 \mathrm{e}-01$ & $9.515467 \mathrm{e}-01$ \\
\hline
\end{tabular}

Table 2: Computational results for the test example. Estimated exponents describing rates of convergence $\mathcal{O}\left(h^{\alpha}().\right)$ of the single components of the proposed error estimate.
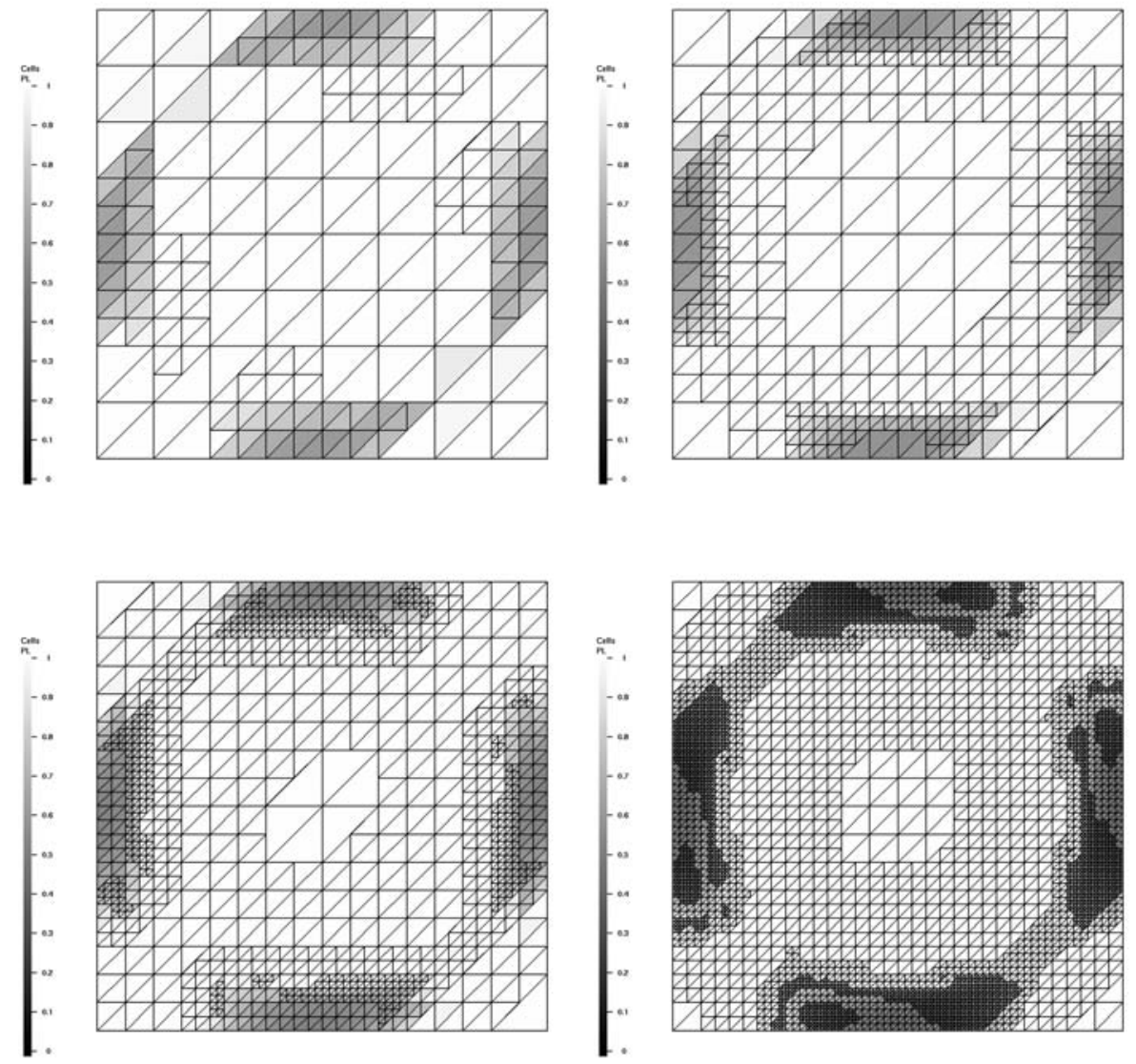

Figure 1: Sequence of locally refined grids produced by our numerical simulation, showing that especially the critical zone where plastification occurs is well resolved. Background colour is given by values of $p^{-1}\left(u_{L}\right)$. 


\section{References}

[1] R. Becker. An Adaptive Finite Element Method for the Incompressible Navier-Stokes Equations on Time-Dependent Domains. Dissertation, Institut für Angewandte Mathematik, Universität Heidelberg, 1995.

[2] A. Bensoussan and J. Frehse. Asymptotic behaviour of the time dependent norton-hoff law in plasticity theory an $h^{1}$ regularity. Comment. Math. Univ. Carilinae, 37(2), 1996.

[3] G. Duvaut and J. L. Lions. Inequalities in Mechanics and Physics. Springer, Berlin-Heidelberg-New York, 1976.

[4] R. Glowinski. Numerical methods for nonlinear variational problems. Springer Series in Comp. Physics. Springer, 1983.

[5] R. Glowinski, J.L. Lions, and R. Trémolières. Numerical Analysis of Variational Inequalities. North-Holland, 1981.

[6] C. Johnson. Existence theorems for plasticity problems. J.Math. Pures Appl., 55:431-444, 1976.

[7] C. Johnson. On Plasticity with Hardening. Journal of math. Analysis and Applications, 62:325-336, 1978.

[8] C. Johnson and P. Hansbo. Adaptive finite element methods for small strain elasto-plasticity. Technical report, Chalmers Univ. of Technology, University Göteborg, 1991.

[9] D. Kinderlehrer and G. Stampacchia. An introduction to variational inequalities and their applications. Academic Press, 1980.

[10] G.A. Seregin. On the regularity of weak solutions of variational problems in plasticity theory. Soviet Math. Dokl., 42(2), 1991.

[11] G. Strang. A minimax problem in plasticity theory. Functional analysis methods in numerical analysis, Spec. Sess., AMS, St. Louis 1977, Lect. Notes Math. 701. 319-333. Springer, 1979.

[12] P.-M. Suquet. Existence et Regularite de Solutions des Equations de la Plasticite parfaite. PhD thesis, Universite Paris Sud, 1978. These de $3^{\text {me }}$ cycle.

[13] F.T. Suttmeier. Adaptive Finite Element Approximation of Problems in Elasto-Plasticity Theory. Dissertation, Institute for Applied Mathematics, University of Heidelberg, 1996.

Received: June, 2012 